\title{
A $\mathrm{SnO}_{\mathrm{x}}$ Quantum Dots Embedded Carbon
}

\section{Nanocage Network with Ultrahigh Li Storage}

\section{Capacity}

Yanan Zhang, ${ }^{1}$ Dong Yan, ${ }^{2}$ Zefei Liu, ${ }^{1}$ Youwen Ye, ${ }^{1}$ Fei Cheng, ${ }^{*, 1,2}$ Huanrong $L i^{*, 1}$ and An-

Hui $L u^{*, 2}$

${ }^{1}$ National-Local Joint Engineering Laboratory for Energy Conservation in Chemical Process Integration and Resources Utilization, Tianjin Key Laboratory of Chemical Process Safety, School of Chemical Engineering and Technology, Hebei University of Technology, Guangrong Road 8, Hongqiao District, Tianjin 300130, P. R. China.

${ }^{2}$ State Key Laboratory of Fine Chemicals, School of Chemical Engineering, Dalian University of Technology, Linggong Road 2, Ganjingzi District, Dalian 116024, P. R. China.

*Corresponding authors: fcheng@hebut.edu.cn;

lihuanrong@hebut.edu.cn; anhuilu@dlut.edu.cn. 


\section{Supporting Figures}
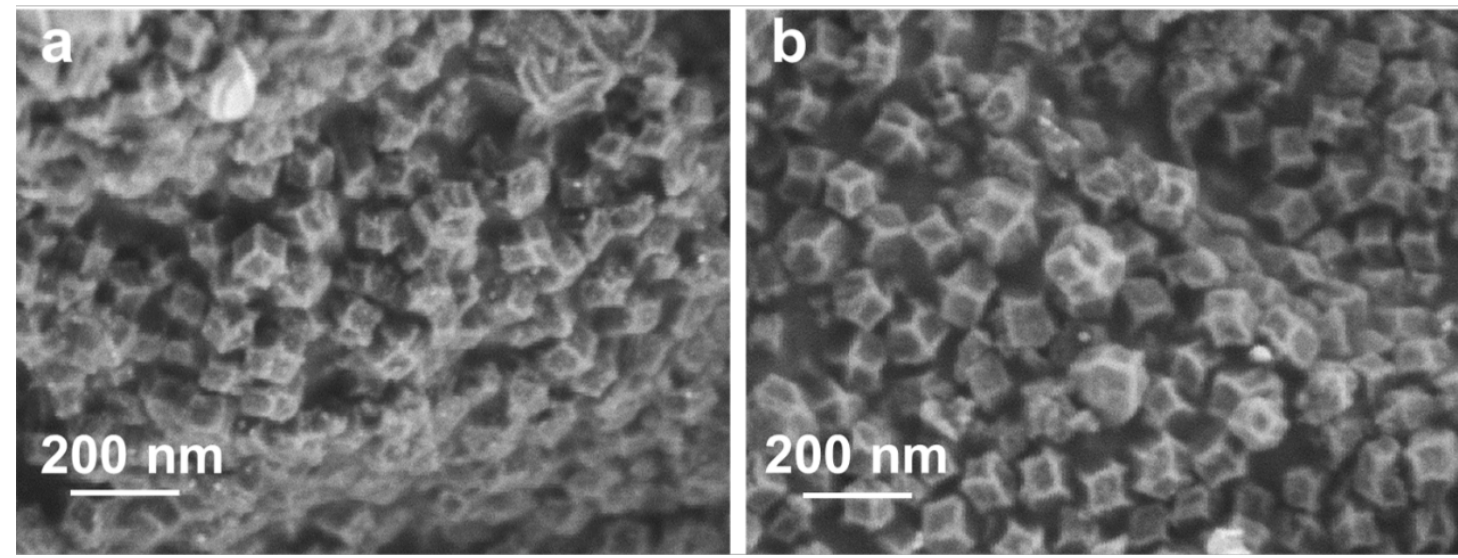

Figure S1. SEM images of the SQD@C hybrids (a) SQD@C-1 and (b) SQD@C-3. 

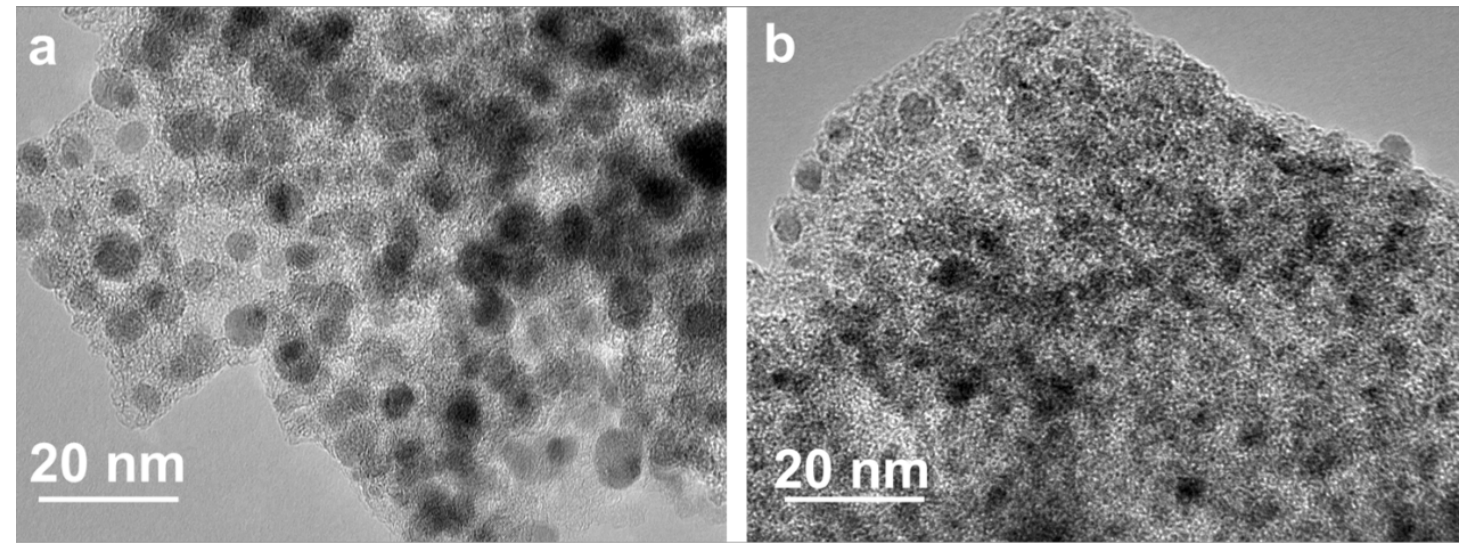

Figure S2. TEM images of the SQD@C hybrids (a) SQD@C-1 and (b) SQD@C-3. 

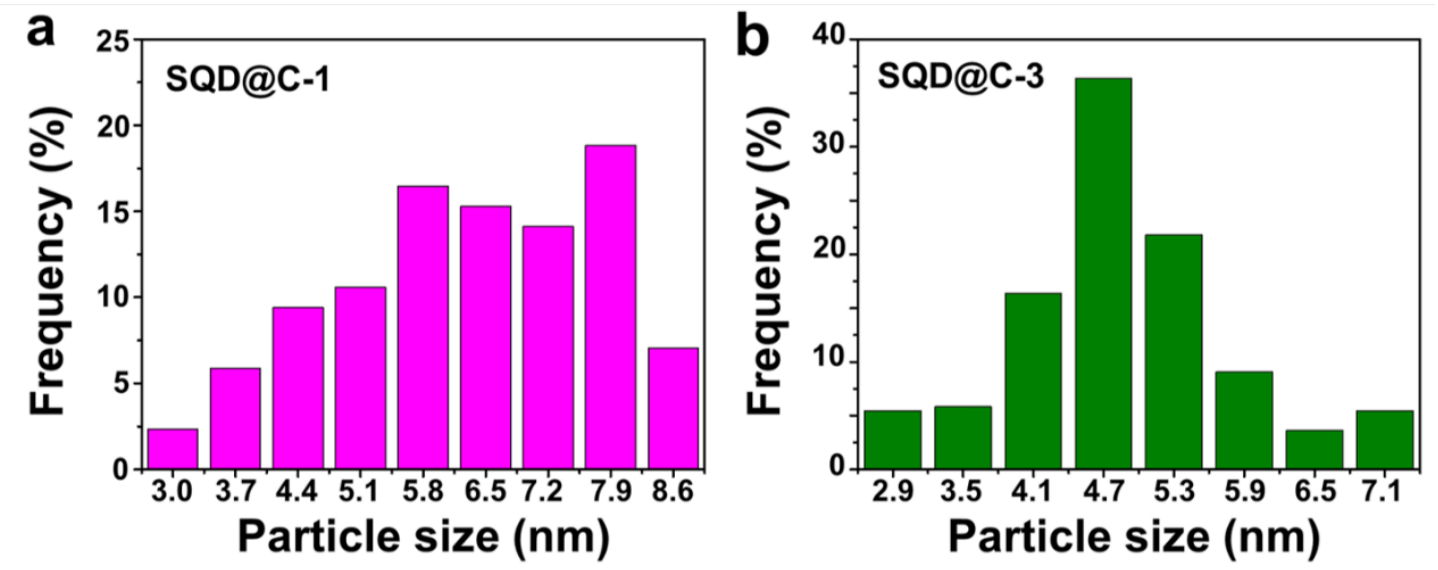

Figure S3. The particle size distribution of $\mathrm{SnO}_{\mathrm{x}}$ quantum dots of (a) SQD@C-1 and (b) SQD@C-3. 

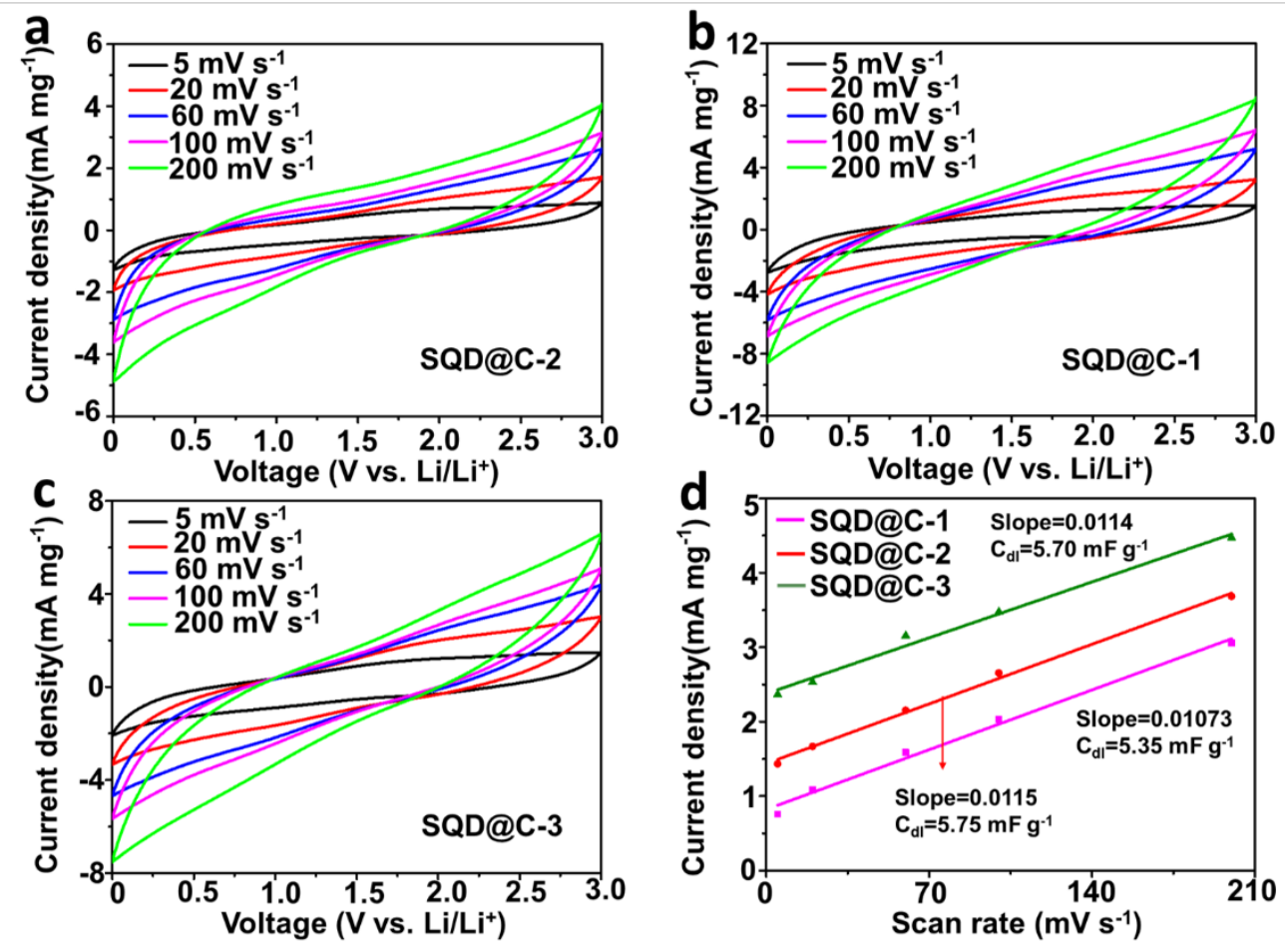

Figure S4. Cyclic voltammograms for (a) SQD@C-1, (b) SQD@C-2, (c) SQD@C-3 measured at different scan rates from 5 to $200 \mathrm{mV} \mathrm{s}^{-1}$ and (d) corresponding plots of the current density at $1.2 \mathrm{~V} v s \mathrm{Li} / \mathrm{Li}^{+}$.

It is proved that the variation tendency of ECSA is consistent with that of $\mathrm{C}_{\mathrm{dl}}$, which can be obtained via cyclic voltammetry $(\mathrm{CV})$ at various scan rates. ${ }^{\mathrm{S} 1}$ Therefore, the CV curves of SQD@C hybrids were measured to calculate the $\mathrm{C}_{\mathrm{dl}}$. Based on the CV profiles at various scan rates over the potential range from 0 to $3 \mathrm{~V}\left(v s . \mathrm{Li} / \mathrm{Li}^{+}\right)$, the difference values of current density $\left(\triangle \mathrm{j}=\mathrm{j}_{\left.\mathrm{a}-\mathrm{j}_{\mathrm{c}}\right)}\right.$ are obtained, which are directly proportional to the scan rates. We plot $\triangle \mathrm{j}$ against different scan rates to acquire the linear fitting line, from which the half slope value $\left(\mathrm{C}_{\mathrm{dl}}\right)$, can be obtained. 


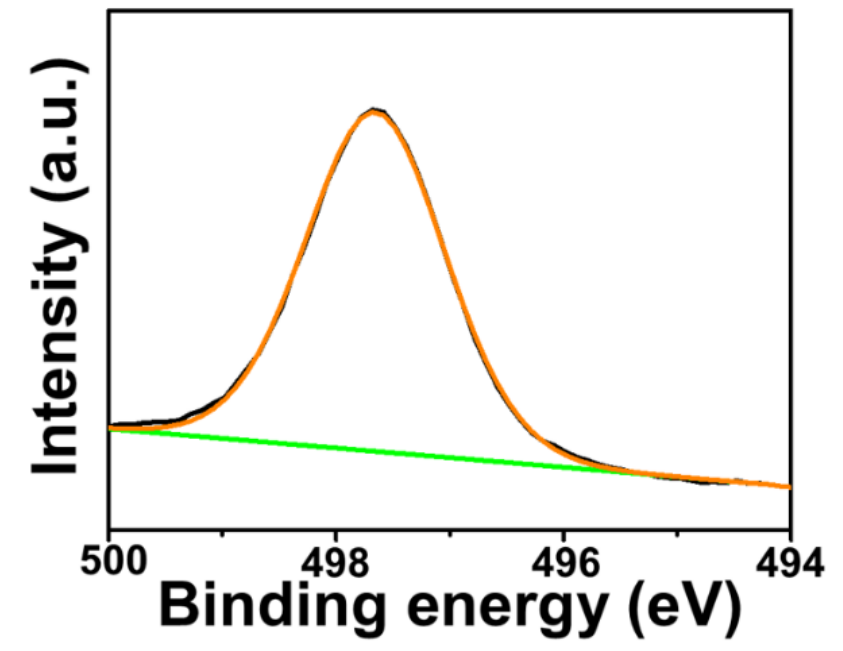

Figure S5. Zn LMM Auger peaks of SQD@C-2. 


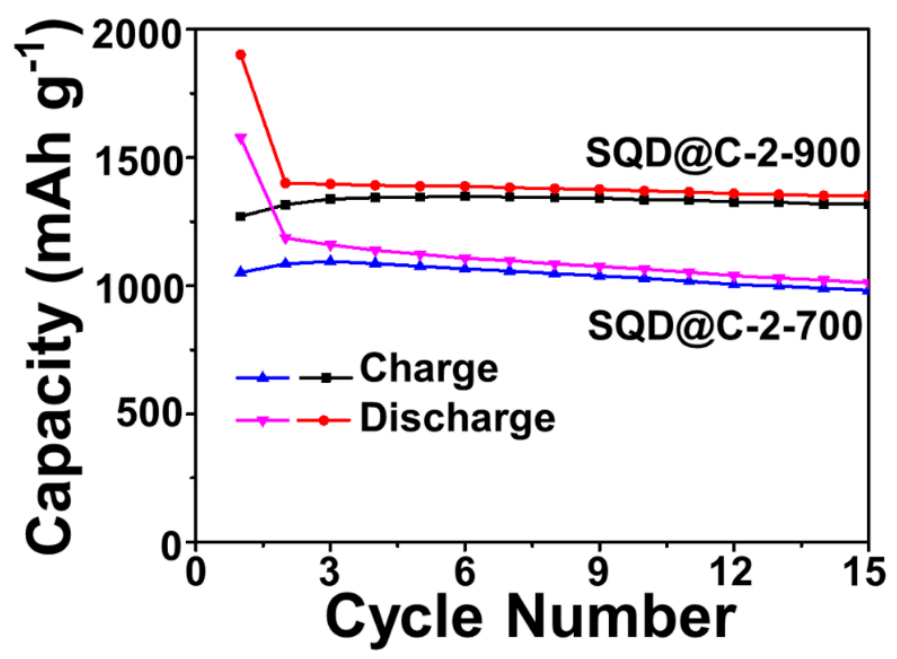

Figure S6. Cycling performances of the samples pyrolyzed at 700 and $900{ }^{\circ} \mathrm{C}$. 

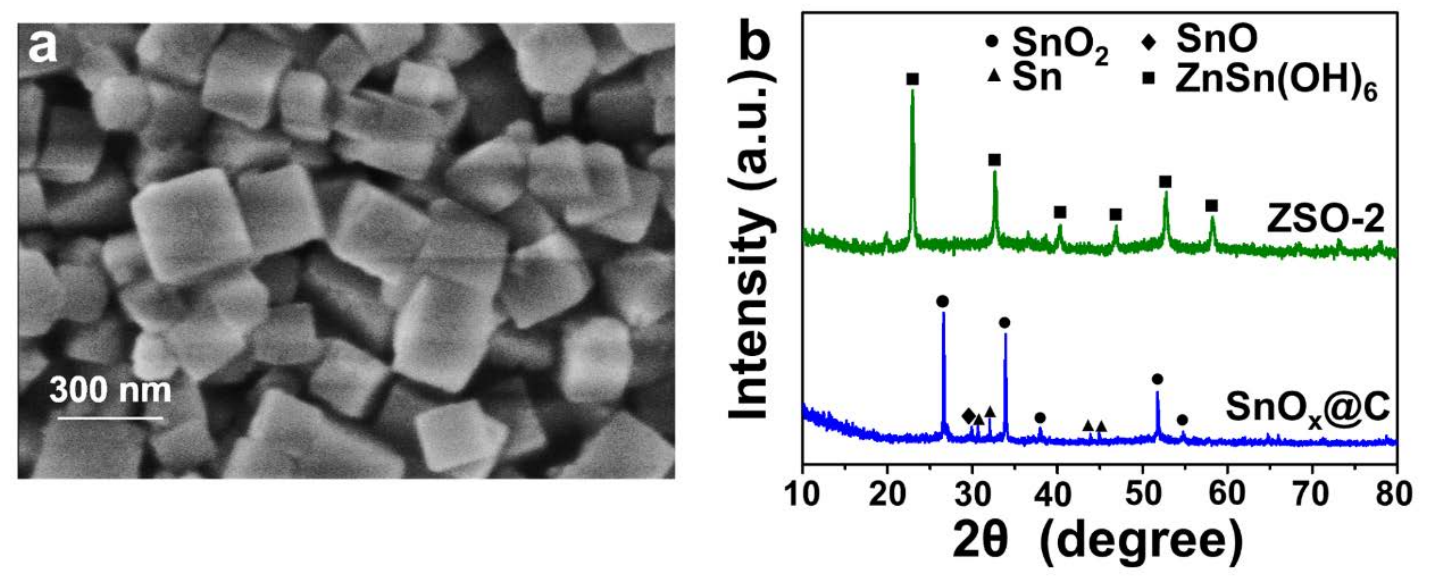

Figure S7. (a) SEM image of $\mathrm{ZnSn}(\mathrm{OH})_{6}$ precursor (ZSO-2); (b) XRD curves of ZSO-2 and SnOx@C. 


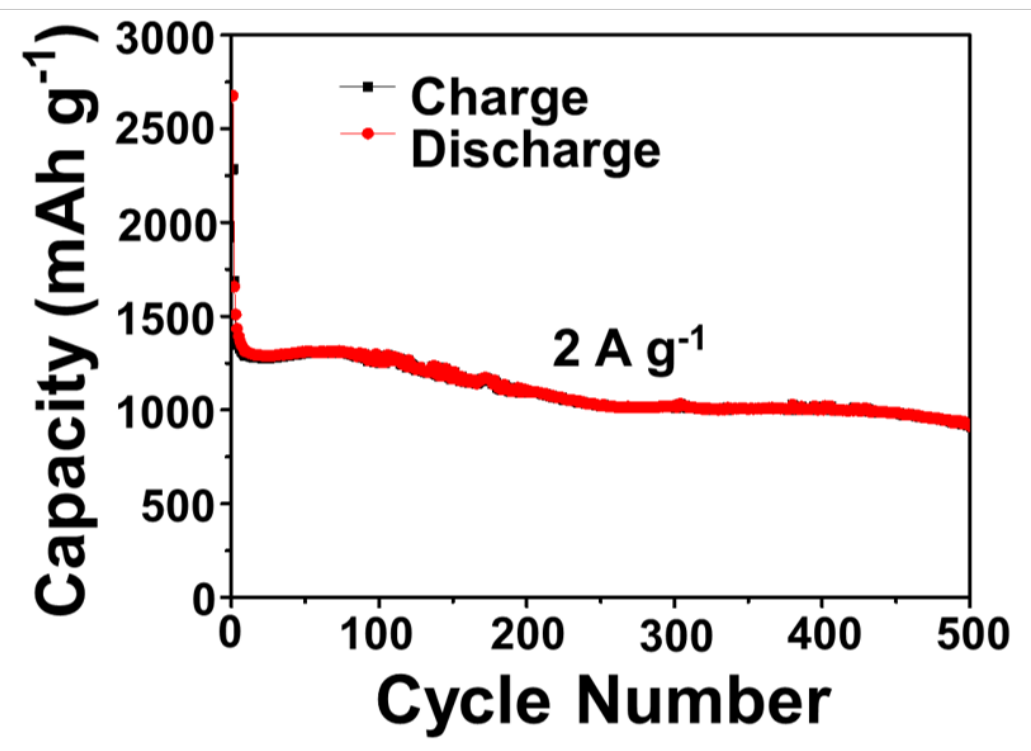

Figure S8. The long cycling performance of SQD@C-2 at $2 \mathrm{Ag}^{-1}$. 


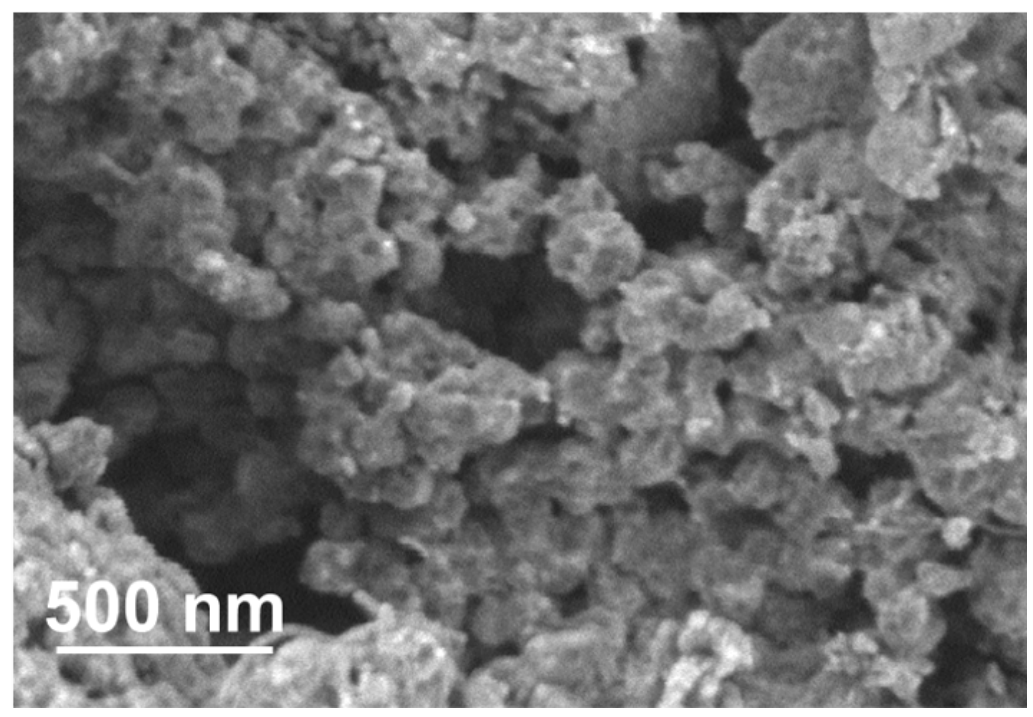

Figure S9. The SEM image of SQD@C-2 after cycling. 


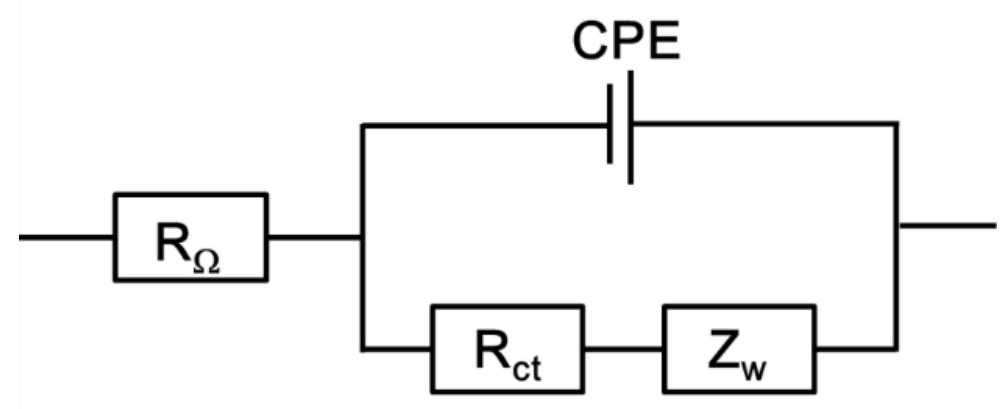

Figure S10. The equivalent circuit models of the SQD@C hybrids.

The calculation of lithium-ion diffusion coefficients: ${ }^{\text {s2 }}$

$$
Z_{w}=R_{c t}+R_{\Omega}+
$$

$\omega^{-1 / 2}$

(1)

The calculation of lithium-ion diffusion coefficient is calculated Eq. (1), in which $\mathrm{R}_{\mathrm{ct}}, \mathrm{R}_{\Omega}$, and $\omega^{-1 / 2}$ are the charge transfer resistance, electrolyte resistance, and angular frequency, respectively. The parameter $\sigma$ is the Warburg factor corresponding to the slope of the curve in Figure 4f, which is related to the lithium ion diffusion coefficient. The diffusion coefficient could be obtained from Eq. (2),

$$
\begin{gathered}
D= \\
\frac{R^{2} T^{2}}{2 A^{2} n^{4} F^{4} C^{2} \sigma^{2}}
\end{gathered}
$$

(2)

where $\mathrm{R}$ represents the gas constant (8.314 approximate), $\mathrm{T}$ is the absolute temperature, $\mathrm{A}$ is the surface area of the cathode, $\mathrm{n}$ is the number of electrons per molecule during oxidization, F the Faraday constant (96485.33 $\mathrm{C} \mathrm{mol}^{-1}$ ), $\mathrm{C}$ the concentration of lithium-ion, and $\sigma$ the Warburg factor associated with $\mathrm{Z}_{\mathrm{w}}$. 


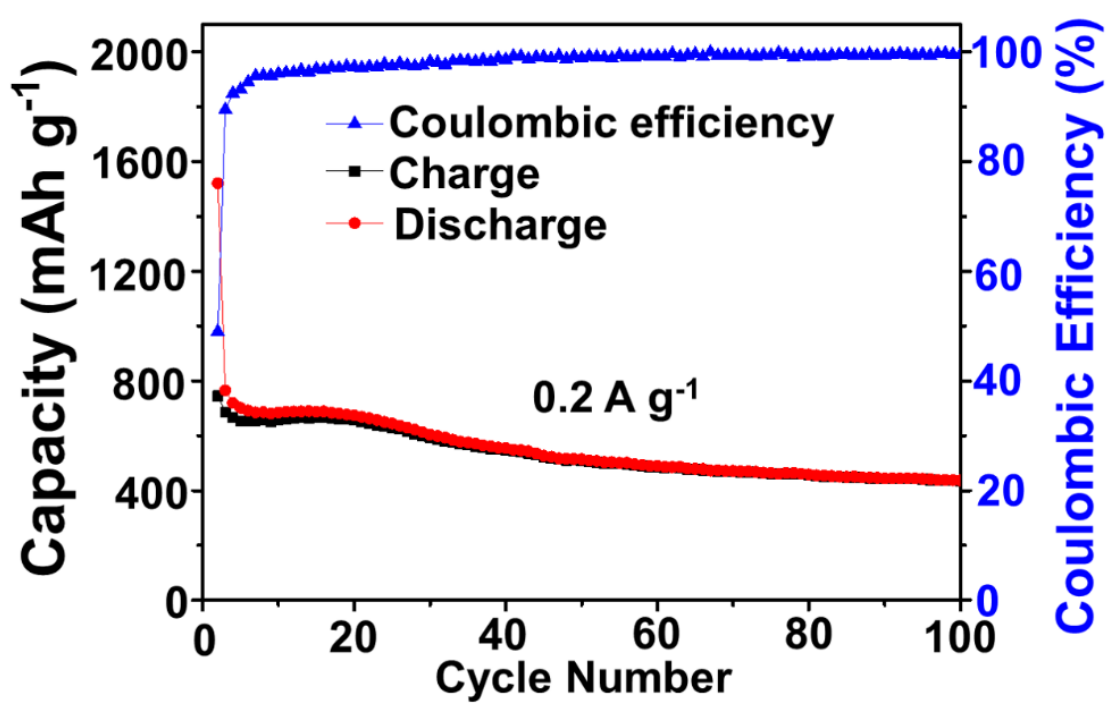

Figure S11. Cycling performance of ZIF-8 derived carbon. 
Table S1. Surface area and pore volume for SQD@C hybrids.

\begin{tabular}{ccc}
\hline Materials & Surface Area $\left(\mathrm{m}^{2} \mathrm{~g}^{-1}\right)$ & Pore Volume $\left(\mathrm{cm}^{3} \mathrm{~g}^{-1}\right)$ \\
\hline SQD@C-1 & 353 & 0.29 \\
SQD@C-2 & 589 & 0.54 \\
SQD@C-3 & 445 & 0.44 \\
\hline
\end{tabular}


Table S2. The fitting values of $\mathrm{R}_{\Omega}, \mathrm{R}_{\mathrm{ct}}, \mathrm{C}$ and $\mathrm{Z}_{\mathrm{w}}$.

\begin{tabular}{ccccc}
\hline & $\mathrm{R}_{\Omega}(\mathrm{ohm})$ & $\mathrm{R}_{\mathrm{ct}}(\mathrm{ohm})$ & $\mathrm{Z}_{\mathrm{w}}\left(\mathrm{S} \mathrm{sec}^{0.5}\right)$ & $\mathrm{C}(\mathrm{F})$ \\
\hline SQD@C-1 & 3.9 & 121.3 & $5.0 \times 10^{-3}$ & $2.2 \times 10^{-5}$ \\
SQD@C-2 & 3.6 & 113.7 & $1.7 \times 10^{-3}$ & $1.7 \times 10^{-6}$ \\
SQD@C-3 & 4.5 & 185.3 & $1.5 \times 10^{-3}$ & $1.4 \times 10^{-6}$ \\
\hline
\end{tabular}


Table S3. Electrochemical performance comparison of some SnOx@C anodes for lithium-ion batteries.

\begin{tabular}{|c|c|c|c|}
\hline $\begin{array}{c}\text { Sample } \\
\text { name }\end{array}$ & $\begin{array}{c}\text { Capacity } \\
\left(\mathrm{mAh} \mathbf{g}^{-1}\right) \\
/ \text { Current } \leq 1 \text { A g }^{-1}\end{array}$ & $\begin{array}{c}\text { Capacity } \\
\left(\mathrm{mAh} \mathbf{g}^{-1}\right) \\
/ \text { Current }>1 \text { A g }^{-1}\end{array}$ & Ref. \\
\hline SQD@C-2 & $\begin{array}{l}1824 \mathrm{mAh} \mathrm{g}^{-1} \\
\left(0.2 \mathrm{~A} \mathrm{~g}^{-1}\right) \\
1588 \mathrm{mAh} \mathrm{g}^{-1} \\
\left(1 \mathrm{~A} \mathrm{~g}^{-1}\right)\end{array}$ & $\begin{array}{l}1408 \mathrm{mAh} \mathrm{g}^{-1} \\
\left(2 \mathrm{~A} \mathrm{~g}^{-1}\right) \\
850 \mathrm{mAh} \mathrm{g}^{-1} \\
\left(5 \mathrm{~A} \mathrm{~g}^{-1}\right)\end{array}$ & This work \\
\hline $\begin{array}{c}\mathrm{SnO}_{2-} \\
\mathrm{Mn} / \text { Graphite }\end{array}$ & $\begin{array}{c}850 \mathrm{mAh} \mathrm{g}^{-1} \\
\left(0.2 \mathrm{~A} \mathrm{~g}^{-1}\right)\end{array}$ & $\begin{array}{c}700 \mathrm{mAh} \mathrm{g}^{-1} \\
\left(2 \mathrm{~A} \mathrm{~g}^{-1}\right)\end{array}$ & S3 \\
\hline $\begin{array}{c}\text { Ferrocene- } \\
\text { SnO }_{2} / \text { graphene }\end{array}$ & $\begin{array}{l}1085 \mathrm{mAh} \mathrm{g}^{-1} \\
\left(0.1 \mathrm{~A} \mathrm{~g}^{-1}\right) \\
752 \mathrm{mAh} \mathrm{g}^{-1} \\
\left(1 \mathrm{~A} \mathrm{~g}^{-1}\right)\end{array}$ & -- & S4 \\
\hline $\begin{array}{c}\mathrm{Sn} / \mathrm{SnO}_{2} @ \mathrm{C} \\
\text { nanofibers }\end{array}$ & $\begin{array}{l}\square 986 \mathrm{mAh} \mathrm{g}^{-1} \\
\left(1 \mathrm{~A} \mathrm{~g}^{-1}\right)\end{array}$ & $\begin{array}{l}712 \mathrm{mAh} \mathrm{g}^{-1} \\
\left(2 \mathrm{~A} \mathrm{~g}^{-1}\right) \\
596 \mathrm{mAh} \mathrm{g}^{-1} \\
\left(4 \mathrm{~A} \mathrm{~g}^{-1}\right)\end{array}$ & S5 \\
\hline $\begin{array}{l}\text { SnO2@N/S- } \\
\text { codoped } \\
\text { graphene }\end{array}$ & $\begin{array}{l}1002 \mathrm{mAh} \mathrm{g}^{-1} \\
\left(0.2 \mathrm{~A} \mathrm{~g}^{-1}\right) \\
893 \mathrm{mAh} \mathrm{g}^{-1} \\
\left(1 \mathrm{~A} \mathrm{~g}^{-1}\right)\end{array}$ & $\begin{array}{l}698 \mathrm{mAh} \mathrm{g}^{-1} \\
\left(2 \mathrm{~A} \mathrm{~g}^{-1}\right) \\
477 \mathrm{mAh} \mathrm{g}^{-1} \\
\left(5 \mathrm{~A} \mathrm{~g}^{-1}\right)\end{array}$ & S6 \\
\hline $\begin{array}{c}\text { porous } \mathrm{SnO}_{2} / \mathrm{C} \\
\text { nanofibers }\end{array}$ & $\begin{array}{l}750 \mathrm{mAh} \mathrm{g}^{-1} \\
\left(0.5 \mathrm{~A} \mathrm{~g}^{-1}\right) \\
685 \mathrm{mAh} \mathrm{g}^{-1} \\
\left(1 \mathrm{~A} \mathrm{~g}^{-1}\right)\end{array}$ & $\begin{array}{l}495 \mathrm{mAh} \mathrm{g}^{-1} \\
\left(3 \mathrm{~A} \mathrm{~g}^{-1}\right) \\
521 \mathrm{mAh} \mathrm{g}^{-1} \\
\left(5 \mathrm{~A} \mathrm{~g}^{-1}\right)\end{array}$ & S7 \\
\hline $\mathrm{SnO}_{2} / \mathrm{NC}$ & $\begin{array}{l}1255 \mathrm{mAh} \mathrm{g}^{-1} \\
\left(0.1 \mathrm{~A} \mathrm{~g}^{-1}\right) \\
836 \mathrm{mAh} \mathrm{g}^{-1} \\
\left(1 \mathrm{~A} \mathrm{~g}^{-1}\right)\end{array}$ & $\begin{array}{l}738 \mathrm{mAh} \mathrm{g}^{-1} \\
\left(2 \mathrm{~A} \mathrm{~g}^{-1}\right) \\
615 \mathrm{mAh} \mathrm{g}^{-1} \\
\left(4 \mathrm{~A} \mathrm{~g}^{-1}\right)\end{array}$ & S8 \\
\hline $\mathrm{SnO}_{2} / \mathrm{MXene}$ & $\begin{array}{c}530 \mathrm{mAh} \mathrm{g}^{-1} \\
\left(1 \mathrm{~A} \mathrm{~g} \mathrm{~g}^{-1}\right)\end{array}$ & $\begin{array}{l}489 \mathrm{mAh} \mathrm{g}^{-1} \\
\left(2 \mathrm{~A} \mathrm{~g}^{-1}\right) \\
310 \mathrm{mAh} \mathrm{g}^{-1} \\
\left(5 \mathrm{~A} \mathrm{~g}^{-1}\right)\end{array}$ & S9 \\
\hline $\begin{array}{c}\mathrm{SnO}_{2} @ \mathrm{MOF} / \\
\text { graphene }\end{array}$ & $\begin{array}{c}450 \mathrm{mAh} \mathrm{g}^{-1} \\
\left(1 \mathrm{~A} \mathrm{~g}^{-1}\right)\end{array}$ & $\begin{array}{c}324 \mathrm{mAh} \mathrm{g}^{-1} \\
\quad\left(2 \mathrm{~A} \mathrm{~g}^{-1}\right)\end{array}$ & S10 \\
\hline
\end{tabular}




\section{Supplementary Information References}

(S1)Wu, H. B.; Xia, B. Y.; Yu, L.; Yu, X.-Y.; Lou, X. W. Porous Molybdenum Carbide NanoOctahedrons Synthesized via Confined Carburization in Metal-Organic Frameworks for Efficient Hydrogen Production. Nat. Commun. 2015, 11, 6512.

(S2) Yang, Y.; Zhao, X.; Wang, H. -E.; Li, M.; Hao, C.; Ji, M.; Ren, S.; Cao, G. Phosphorized $\mathrm{SnO}_{2} /$ Graphene Heterostructure for Highly Reversible Lithium-Ion Storage with Enhanced Pseudocapacitance. J. Mater. Chem. A 2018, 6, 3479-3487.

(S3) Hu, R.; Ouyang, Y.; Liang, T.; Tang, X.; Yuan, B.; Liu, J.; Zhang, L.; Yang, L.; Zhu, M. Inhibiting Grain Coarsening and Inducing Oxygen Vacancy: The Roles of $\mathrm{Mn}$ in Achieving Highly Reversible Conversion Reaction and Long Life $\mathrm{SnO}_{2}-\mathrm{Mn}$-Graphite Ternary Anode. Energy Environ. Sci. 2017, 10, 2017-2029.

(S4) Zhang, S.; Liang, B.; Fan, Y.; Wang, J.; Liang, X.; Huang, H.; Huang, D.; Zhou, W.; Guo, J. Ferrocene as a Novel Additive to Enhance the Lithium-Ion Storage Capability of $\mathrm{SnO}_{2} /$ Graphene Composite. ACS Appl. Mater. Interfaces 2019, 11, 31943-31953.

(S5) Gao, S.; Wang, N.; Li, S.; Li, D.; Cui, Z.; Yue, G.; Liu, J.; Zhao, X.; Jiang, L.; Zhao, Y. Multi-Wall Sn/SnO ${ }_{2} @$ Carbon Hollow Nanofibers Anode Material for High-Rate and Long-Life Lithium-Ion Batteries. Angew. Chem. Int. Ed. 2020, 59, 2465-2472.

(S6) Wang, H.-G.; Wu, Q.; Wang, Y.; Wang, X.; Wu, L.; Song, S.; Zhang, H. Molecular Engineering of Monodisperse $\mathrm{SnO}_{2}$ Nanocrystals Anchored on Doped Graphene with High-Performance Lithium/Sodium-Storage Properties in Half/Full Cells. Adv. Energy Mater. 2019, 9, 1802993.

(S7) Kwon, O. H.; Oh, J. H.; Gu, B.; Jo, M. S.; Oh, S. H.; Kang, Y. C.; Kim, J.-K.; Jeong, S. M.; Cho, J. S. Porous $\mathrm{SnO}_{2} / \mathrm{C}$ Nanofiber Anodes and $\mathrm{LiFePO}_{4} / \mathrm{C}$ Nanofiber Cathodes with a Wrinkle Structure for Stretchable Lithium Polymer Batteries with High 
Electrochemical Performance. Adv. Sci. 2020, 7, 2001358.

(S8) Cheng, Y.; Wang, S.; Zhou, L.; Chang, L.; Liu, W.; Yin, D.; Yi, Z.; Wang, L. $\mathrm{SnO}_{2}$ Quantum Dots: Rational Design to Achieve Highly Reversible Conversion Reaction and Stable Capacities for Lithium and Sodium Storage. Small 2020, 16, 2000681.

(S9) Liu, Y.-T.; Zhang, P.; Sun, N.; Anasori, B.; Zhu, Q.-Z.; Liu, H.; Gogotsi, Y.; Xu, B. SelfAssembly of Transition Metal Oxide Nanostructures on MXene Nanosheets for Fast and Stable Lithium Storage. Adv. Mater. 2018, 30, 1707334.

(S10) Gao, C.; Jiang, Z.; Wang, P.; Jensen, L. R.; Zhang, Y.; Yue, Y. Optimized Assembling of $\mathrm{MOF} / \mathrm{SnO}_{2} / \mathrm{Graphene} \mathrm{Leads} \mathrm{to} \mathrm{Superior} \mathrm{Anode} \mathrm{for} \mathrm{Lithium} \mathrm{Ion} \mathrm{Batteries.} \mathrm{Nano} \mathrm{Energy}$ 2020, 74, 104868. 